\title{
IDH-1 deficiency induces growth defects and metabolic alterations in GSPD-1-deficient Caenorhabditis elegans
}

\author{
Hung-Chi Yang ${ }^{1} \cdot$ Hsiang $\mathrm{Yu}^{2} \cdot$ You-Cheng Liu ${ }^{3} \cdot$ Tzu-Ling Chen ${ }^{3} \cdot$ Arnold Stern $^{4} \cdot$ Szecheng J. Lo ${ }^{5}$. \\ Daniel Tsun-Yee Chiu ${ }^{2,3,6,7,8}$
}

Received: 14 August 2018 / Revised: 10 December 2018 / Accepted: 21 December 2018 / Published online: 19 January 2019

(C) The Author(s) 2018

\begin{abstract}
NADPH is a reducing equivalent that maintains redox homeostasis and supports reductive biosynthesis. Lack of major NADPHproducing enzymes predisposes cells to growth retardation and demise. It was hypothesized that double deficiency of the NADPHgenerating enzymes, GSPD-1 (Glucose-6-phosphate 1-dehydrogenase), a functional homolog of human glucose-6-phosphate dehydrogenase (G6PD), the rate-limiting enzyme of the pentose phosphate pathway, and IDH-1 (isocitrate dehydrogenase-1) affect growth and development in the nematode, Caenorhabditis elegans (C. elegans). The idh-1;gspd-1(RNAi) double-deficient C. elegans model displayed shrinkage of body size, growth retardation, slowed locomotion, and impaired molting. Global metabolomic analysis was employed to address whether or not metabolic pathways were altered by severe NADPH insufficiency by the idh-1;gspd-1(RNAi) double-deficiency. The principal component analysis (PCA) points to a distinct metabolomic profile of idh-1;gspd-1(RNAi) doubledeficiency. Further metabolomic analysis revealed that NADPH-dependent and glutamate-dependent amino acid biosynthesis were significantly affected. The reduced pool of amino acids may affect protein synthesis, as indicated by the absence of NAS-37 expression during the molting process. In short, double deficiency of GSPD-1 and IDH-1 causes growth retardation and molting defects, which are, in part, attributed to defective protein synthesis, possibly mediated by altered amino acid biosynthesis and metabolism in C. elegans.
\end{abstract}

Keywords C. elegans $\cdot$ GSPD- $1 \cdot$ IDH-1 $\cdot$ Development $\cdot$ Molting $\cdot$ Metabolomic $\cdot$ Amino acid

\section{Introduction}

Glucose-6-phosphate dehydrogenase (G6PD) is the first and rate-limiting enzyme in the hexose monophosphate shunt
(HMS), also known as the pentose phosphate pathway (PPP). The classical biochemical role of G6PD is to catalyze the oxidation of glucose-6-phosphate to 6-phosphogluconolactone and concomitantly produce the reduced form of nicotinamide
Hung-Chi Yang and Hsiang Yu contributed equally to this work.

Electronic supplementary material The online version of this article (https://doi.org/10.1007/s00109-018-01740-2) contains supplementary material, which is available to authorized users.

Daniel Tsun-Yee Chiu dtychiu@mail.cgu.edu.tw

1 Department of Medical Laboratory Science and Biotechnology, Yuanpei University of Medical Technology, Hsinchu, Taiwan

2 Department of Medical Biotechnology and Laboratory Sciences, College of Medicine, Chang Gung University, Taoyuan, Taiwan

3 Graduate Institute of Biomedical Sciences, College of Medicine, Chang Gung University, Taoyuan, Taiwan

4 New York University School of Medicine, New York, NY, USA
5 Department of Biomedical Sciences, College of Medicine, Chang Gung University, Taoyuan, Taiwan

6 Research Center for Chinese Herbal Medicine, College of Human Ecology, Chang Gung University of Science and Technology, Taoyuan, Taiwan

7 Department of Pediatric Hematology/Oncology, Linkou Chang Gung Memorial Hospital, Taoyuan, Taiwan

8 Healthy Aging Research Center, Chang Gung University, Taoyuan, Taiwan 
adenine dinucleotide phosphate (NADPH) for antioxidant defense and reductive biosynthesis $[1,2]$. G6PD deficiency is the most common enzymopathy affecting 400 million people in the world. Most G6PD mutations are point mutations causing amino acid substitution and reduced enzyme activity. Classically, G6PD deficiency has been linked to red blood cell disorders as the clinical manifestations. Novel functions of G6PD in cellular physiology have been discovered over recent years [3]. G6PD is required for life as severe deficiency of G6PD in mammals and nematodes is lethal for survival and reproduction $[4,5]$ and plays an important role in embryogenesis [6, 7].

The biological importance of G6PD is largely attributed to the production of NADPH. The reducing equivalent NADPH serves pivotal roles in cellular biology. It maintains cellular redox homeostasis by regenerating reduced glutathione, which is needed for detoxifying elevated oxidants. NADPH is also required for reductive biosynthesis of building blocks. Intracellular NADPH is supported by parallel pathways that are localized in different cellular compartments [8]. In the cytoplasm, the main source of NADPH is the oxidative branch of the PPP. Alternative NADPH-producing pathways include cytosolic malic enzyme (ME1), cytosolic and mitochondrial isocitrate dehydrogenase (IDH1 and IDH2), transhydrogenase (NNT), and cytosolic and mitochondrial methylene tetrahydrofolate dehydrogenase (MTHFD1 and MTHFD2) [9]. Understanding how compartmentalization of NADPH homeostasis affecting cell development in health and diseases presents a major investigative challenge.

The biochemical role of IDHs is the oxidation of isocitrate to oxalosuccinate followed by the decarboxylation and ultimately the production of alpha-ketoglutarate, which is coupled with the reduction of $\mathrm{NADP}^{+}$to NADPH. In humans, IDH1 and IDH2 are $\mathrm{NADP}^{+}$-dependent enzymes, while IDH3 is a NAD ${ }^{+}$-dependent multi-subunit mitochondrial enzyme. Human IDH1 localizes in the cytoplasm and peroxisome and is highly expressed in the liver. Human IDH2, which has the mitochondrial signal peptide at the amino terminus, localizes in mitochondria and is highly expressed in mammalian muscle, heart, and lymphocyte [10]. IDH1 is associated with lipid metabolism and glucose sensing [11, 12], whereas IDH2 regulates oxidative respiration [13]. In Caenorhabditis elegans (C. elegans), IDH-1 is predicted as a cytosolic enzyme, whereas IDH-2 is predicted as a mitochondrial enzyme [14]. Although the enzymatic activity of IDH1 has been discovered decades ago, the biological function of wild type (WT) IDH1 is still controversial. Compared to WT IDH1, oncogenic IDH1/2 mutants are well studied and received considerable attention. IDH1/2 mutations have been found in glioma, glioblastoma, acute myelogenous leukemia, chondrosarcoma, and enchondroma $[15,16]$. Animal study shows that IDH null mice are healthy and fertile at steady state, while the liver displays altered amino acid utilization $[17,18]$.
How severe NADPH insufficiency, as a consequence of impairment of different NADPH-producing systems, affects metabolic networks and links to pathophysiology of an organism has not been defined. The notion that NADPH is indispensable for life is supported by the fact that knockdown of NADPH-producing enzymes leads to growth arrest and cell demise. G6PD-deficient human foreskin fibroblasts display slow growth and early onset of senescence [19]. A yeast model with co-disruption of major NADPH sources (G6PD and cytosolic IDH) undergoes growth inhibition and loses viability [20]. Whereas loss of G6PD or IDH alone fails to induce a growth defect, a NADPH compensatory mechanism at the cellular level may maintain NADPH homeostasis and warrant normal development. However, the contribution from each NADPH-producing system at the organismal level remains unclear.

Further study of NADPH-producing systems by applying metabolomic technology to gspd-1 (G6PD homolog) and $i d h$ 1 double-deficient $C$. elegans model will help in clarifying the role of redox homeostasis and regulation in growth and development. Metabolomics is a novel platform of systems biology that aims to characterize all small molecule metabolites (metabolome) in various forms of biological samples. It is a powerful tool to most closely reflect phenotypic expression and it acutely pinpoints the perturbations within metabolic networks. Such metabolic disturbances can be attributed to downstream alterations of genomic and proteomic outcomes. Current advances place metabolomics in the armamentarium of cutting-edge strategies to dissect the metabolic networks of human and animal models in health and diseases. The intermediary metabolic network is conserved among eukaryotic organisms. The nematode $C$. elegans has orthologs for most human metabolic enzymes, including G6PD and IDH1 [21]. C. elegans is a simple and ideal biological system to model human metabolic disturbances. A number of C. elegans studies have taken advantage of different metabolomic approaches, including nuclear magnetic resonance (NMR) spectroscopy, gas/liquid chromatography-coupled mass spectrometry (GC/LC-MS) for analyzing the metabolic pathways in a whole worm [22-28]. Lipidomics has been employed in characterizing the molecular pathway in gspd-1(RNAi) C. elegans. Embryonic lethality occurs through the induction of lipid oxidative damage and activation of lipid-modifying enzymes as identified by lipidomics [6].

In the current study, a global metabolomic platform has been employed for analyzing the metabolome of $i d h-1 ; g s p d-$ 1(RNAi) double-deficient $C$. elegans. Phenotypic characterization has shown that several developmental impairments are found in this double mutant, including defective molting and reduced growth (small body size, delayed growth, and slowed locomotion). The global metabolomic study has also shown that several amino acid metabolic pathways are altered, most notably in those amino acids requiring NADPH for their 
synthesis and metabolism, such as the biosynthesis of valine, leucine, isoleucine; phenylalanine, tyrosine, and tryptophan biosynthesis as well as the metabolism of glutamine and glutamate. These findings enhance our understanding of the causal relationship between insufficient NADPH supply, altered amino acid metabolism, and the resulting developmental defects.

\section{Results}

\section{Growth retardation and molting defect of idh-1;gspd-1(RNAi) double-deficient C. elegans}

Mutants of isocitrate dehydrogenases were used to investigate the complementary roles of cytoplasmic NADPH-producing pathways during growth and development. In brief, gspd-1 RNAi was used in $i d h-1$ and $i d h-2$ deletion mutants to generate idh-1;gspd-1(RNAi) and idh-2;gspd-1(RNAi) double-deficient C. elegans, respectively (Supplementary Fig. S1). After 72 h, idh-1;gspd-1(RNAi) double-deficient C. elegans, compare to controls including Mock, gspd-1(RNAi), idh-1 and idh-2 mutant as well as $i d h-1(R N A i)$; idh-2 and $i d h-1 ; i d h-2(R N A i)$ double-deficient $C$. elegans, displayed shrinkage of body size (Fig. 1a). The idh-1;gspd-1(RNAi) double-deficient $C$. elegans also showed growth retardation (Supplementary Fig. S2) and slowed locomotion (Supplementary Fig. S3). The body size of idh-1;gspd-1(RNAi) double-deficient C. elegans was significantly decreased $(P<0.05)$ as determined by the perimeter and area measurements (Fig. 1b, c). Growth defect was not observed in gspd-1(RNAi) C. elegans. Neither the idh-1 nor the $i d h-2$ mutation affected $C$. elegans growth. Likewise, $i d h$ 2;gspd-1(RNAi) double-deficient $C$. elegans had no reduction in body size.

The idh-1;gspd-1(RNAi) double-deficient C. elegans displayed an abnormal molting process, which was not observed in Mock, gspd-1(RNAi), and idh-1 mutant C. elegans. Such an abnormal molting process is demonstrated in Fig. 2a showing the molting defects at the head and Fig. $2 b$ showing the molting defects at the tail. Gspd-1 and idh-1 suppression results in a disruption of normal molting indicating that gspd-1 and $i d h-1$ are complementary to each other.

\section{Reduced NAS-37 protease expression in idh-1;gspd-1(RNAi) double-deficient $C$. elegans}

Molting is critical for transition between larval stages in nematodes. In C. elegans, three key steps, including apolysis (breaking the connection between old cuticle to the hypoderm), cuticle synthesis, and ecdysis (shedding the old cuticle when new cuticle is formed), must be precisely executed in order to grow and survive. Isolation of the mutations responsible for defective ecdysis identifies that nas-37 is responsible for such a phenotype [29]. The idh-1;gspd-1(RNAi) double-deficient $C$. elegans (Fig. 2a) phenocopied the ecdysis mutants in which the cuticle cannot be shed. The nas-37::gfp fusion reporter strain of NAS-37 protease was used to determine whether or not the protein expression was affected during the molting process [29]. The expression level of NAS$37::$ GFP in all tested C. elegans was unaffected at late L3 (Fig. 3a). At late L4, the NAS-37::GFP signal of $i d h-1 ;$;spd1(RNAi) double-deficient $C$. elegans was reduced, compared with Mock, gspd-1(RNAi), and idh-1 mutant C. elegans (70\% lower than that of Mock C. elegans, $P<0.05)$ (Fig. 3b). Consistently, reduced NAS-37::GFP expression level of $i d h$ 1;gspd-1(RNAi) double-deficient C. elegans at late L4 was found both at $25^{\circ} \mathrm{C}$ (Fig. 3) and $20^{\circ} \mathrm{C}$ (Supplementary Fig. $\mathrm{S} 5$ ). This indicates that sufficient NADPH derived from either GSPD-1 or IDH-1 or both is essential for NAS-37 protein expression to maintain normal molting at late L4 in C. elegans.

\section{Metabolomic amino acid analysis in idh-1;gspd-1(RNAi) double-deficient C. elegans}

Since decreased protein expression could be, in part, attributed to impaired amino acid metabolism, the metabolomics technique was employed to profile metabolomes of $i d h-1 ; g s p d-$ 1(RNAi) double-deficient C. elegans compared to controls. Idh-1;gspd-1(RNAi) double-deficient C. elegans exhibited distinct metabolic abnormalities as indicated by metabolomics analyses. The principal component analysis (PCA) model showed that while Mock control, gspd-1(RNAi), and idh-1deficient $C$. elegans were clustered together, idh-1;gspd1(RNAi) double-deficient C. elegans was separated from the rest of the groups regardless of ESI positive or negative modes (Fig. 4a). The altered metabolites found in idh-1;gspd1(RNAi) double-deficient C. elegans were selected in the ESI positive mode (90 metabolites out of 621 candidates) and in the ESI negative mode (34 metabolites out of 223 candidates). Fourteen metabolites of the ESI positive mode and ten metabolites of the ESI negative mode were identified by searching these selected metabolites against our metabolite database $[30,31]$. Based on the pathway analysis, the metabolic pathways of several amino acids were found to be greatly affected. These amino acids included valine, leucine, isoleucine, phenylalanine, tyrosine and tryptophan biosynthesis, glutamine, glutamate, phenylalanine, arginine, and proline metabolism (Fig. 4b).

Quantitative analysis indicated that several amino acids from $i d h-1 ; g s p d-1(R N A i)$ double-deficient $C$. elegans were significantly reduced compared with Mock (Fig. 5a-c). The glutamate level of $i d h-1 ; g s p d-1(R N A i)$ double-deficient $C$. elegans decreased significantly to $22 \%$ of mock $(P<0.001)$ (Fig. 5a). The level of tryptophan was reduced to $63 \%$ in the idh-1;gspd1(RNAi) double-deficient $C$. elegans compared with that of Mock $(P<0.05)$ (Fig. 5b), whereas the level of tryptophan of 
a
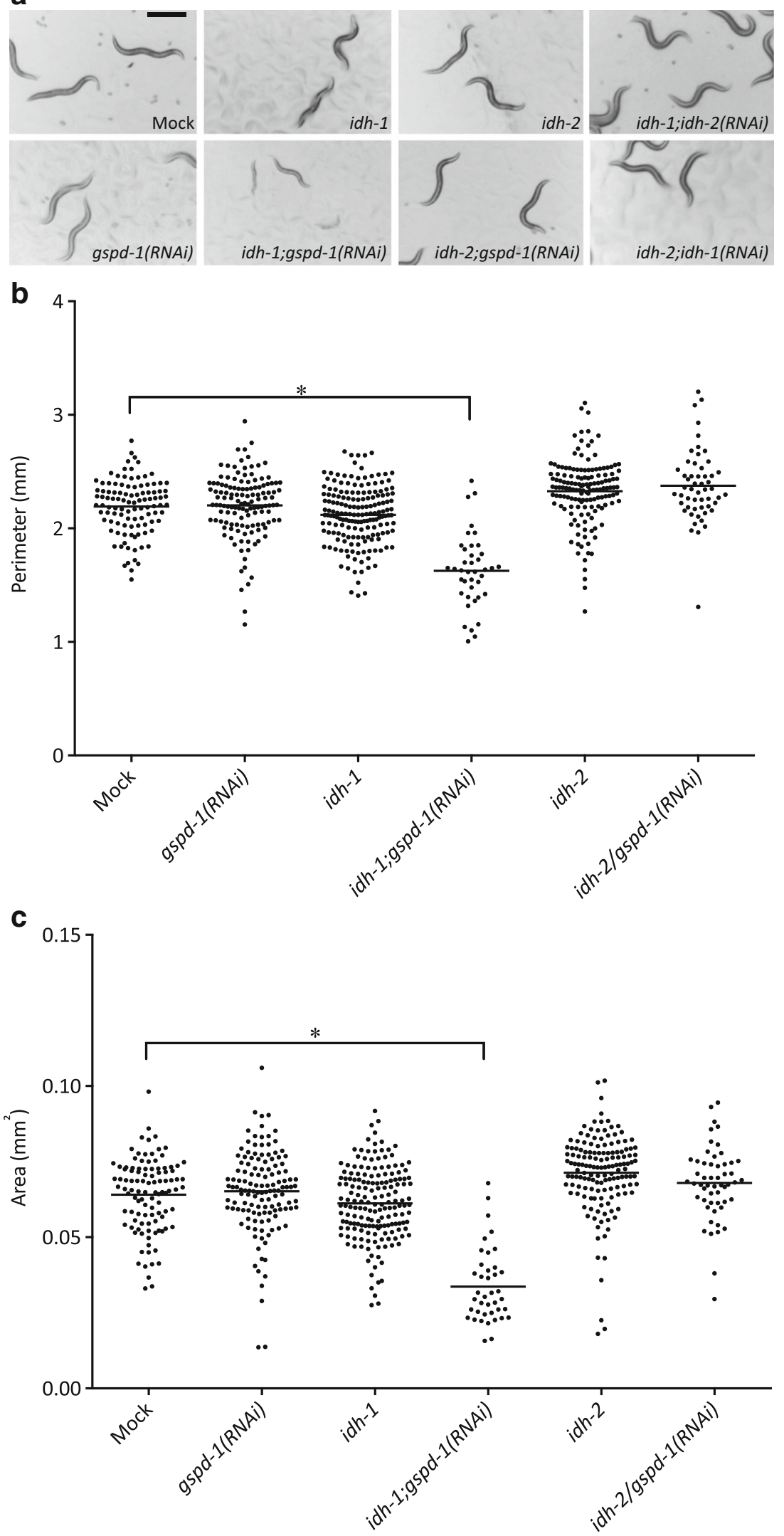
Fig. 1 Decreased body size of $i d h-1 ; g s p d-1$ (RNAi) double-deficient C. elegans compared to mock and other controls. (a) The size of $i d h$ 1;gspd-1(RNAi) double-deficient C. elegans was decreased compared to other $C$. elegans strains at $72 \mathrm{~h}$. Adult $C$. elegans were examined by image analysis software under dissecting microscope. Idh-1;gspdI(RNAi) double-deficient $C$. elegans showed decreased perimeter (b) and area (c) compared to other $C$. elegans strains at $72 \mathrm{~h}$. Each dot represented one adult worm. Horizontal line represented the mean of each C. elegans strain. The black scale bar represented $0.5 \mathrm{~mm}(n \geq 40$, $* P<0.05)$

gspd-1(RNAi) or idh-1 mutant $C$. elegans showed no difference compared with Mock. Phenylalanine and tyrosine levels were reduced in idh-1;gspd-1(RNAi) double-deficient C. elegans compared with Mock (41\% and 34\% of mock, respectively, $P<0.001$ ), while they were unchanged in idh-1 mutant C. elegans, and gspd-1(RNAi) C. elegans (Fig. 5b). The biosynthesis of valine, leucine, and isoleucine was reduced in $i d h$ 1;gspd-1(RNAi) double-deficient $C$. elegans compared with Mock (34\% of mock, $P<0.005 ; 22 \%$ of mock, $P<0.001$; $26 \%$ of mock, $P<0.001$, respectively) (Fig. 5c). The level of arginine and proline metabolism was decreased in idh-1;gspd1(RNAi) double-deficient mutant compared with Mock (39\% of mock, $P<0.005$ and $37 \%$ of mock, $P<0.001$, respectively) (Fig. 5d).

\section{Discussion}

Embryos derived from $C$. elegans fed with Escherichia coli expressing RNA-mediated interference (RNAi)-targeting gspd-1 gene display developmental defects in their embryos, including hatching, membrane function, and eggshell structure $[5,6]$. No defect in larval development is observed in the first generation of gspd-1(RNAi) C. elegans compared to control C. elegans. Perhaps, residual GSPD-1 activity and another NADPH-producing system can generate sufficient NADPH, in gspd-1(RNAi) C. elegans, to meet the basic requirement for larval growth until the reproduction period. It is not clear how IDH-1, another NADPH-producing system, can affect growth and development in GSPD-1 deficiency.
In the current study, the small body size of idh-1;gspd1(RNAi) double-deficient $C$. elegans is consistent with a previous report [32]. Similar findings are seen in yeast and nematode models lacking both G6PD and IDH [20, 32]. The distinct metabolomic profile of idh-1;gspd-1(RNAi) doubledeficient $C$. elegans suggests that severe NADPH insufficiency causes a major disturbance in metabolism and is linked to its defective phenotypes, including reduced body size and impaired molting in $C$. elegans. It is speculated that the compensation of the complementary NADPH-producing systems supports reductive biosynthesis and provides sufficient reducing power to meet the need of cells (Fig. 6a).

Essential amino acids, including arginine, histidine, isoleucine, leucine, lysine, methionine, phenylalanine, threonine, tryptophan, and valine, are required to support the growth of C. elegans [21, 33]. The global metabolomic analysis revealed that amino acid biosynthesis and metabolism were significantly affected by the diminution of both GSPD-1 and IDH-1 in $C$. elegans. Since glutamate dehydrogenase requires $\mathrm{NADPH}$ to synthesize glutamate from $\alpha$ ketoglutarate and $\mathrm{NH}_{4}{ }^{+}$, the level of glutamate was decreased in idh-1;gspd-1(RNAi) double-deficient C. elegans. Glutamate is a precursor of the non-essential amino acids, namely proline and arginine [21], which were reduced in idh-1;gspd-1(RNAi) deficient C. elegans. The essential branched chain amino acids, such as valine, leucine, and isoleucine, were decreased in idh-1;gspd-1(RNAi) doubledeficient $C$. elegans due to the requirement of NADPH for the biosynthesis of these amino acids. In addition, the levels of tryptophan and phenylalanine were decreased in idh-1;gspd-1(RNAi) double-deficient $C$. elegans. The fact that decreased amino acid pool found in idh-1;gspd-1(RNAi) doubledeficient $C$. elegans raises a question whether supplementation with amino acids rescues the growth defect. Preliminary study to address this issue is already being undertaken by our group; however, more detailed works are needed (Supplementary text). Taken together, decreased amino acids found in idh-1;gspd-1(RNAi) double-deficient C. elegans reiterate the importance of the NADPH supply in reductive biosynthesis during organismal development (Fig. 6a).
Fig. 2 Molting defect of $i d h$ 1;gspd-1(RNAi) double-deficient C. elegans compared to mock and other controls. Idh- 1 ; gspdI(RNAi) double-deficient C. elegans showed a molting defect at the L4/Adult stage. Head (a) and tail (b) cuticle of C. elegans cultured at $20^{\circ} \mathrm{C}$ for $54 \mathrm{~h}$ was photographed using a DIC microscope a

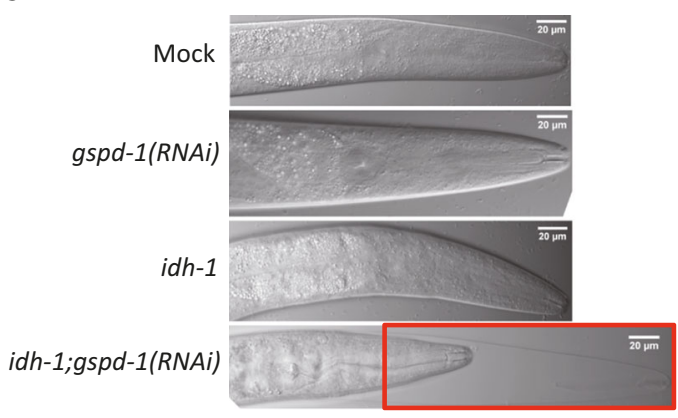

b

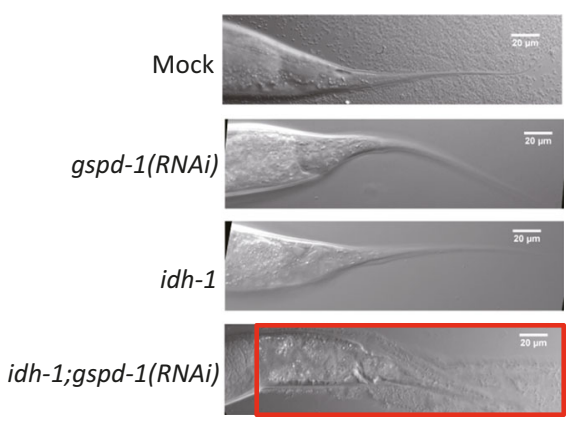


Fig. 3 Decreased molting protein expression of $i d h-1 ; g s p d-1(R N A i)$ double-deficient $C$. elegans compared to controls. Idh-1;gspdl(RNAi) double-deficient C. elegans showed decreased molting protein NAS-37::GFP expression $3 \mathrm{~h}$ before the L4/adult molting. a NAS-37::GFP expression of $C$. elegans cultured at $25^{\circ} \mathrm{C}$ for $28 \mathrm{~h}(3 \mathrm{~h}$ before $\mathrm{L} 3 / \mathrm{L} 4$ molting) and $34 \mathrm{~h}$ ( $3 \mathrm{~h}$ before L4/ adult molting) was photographed using a fluorescence microscope. The arrow points to the position of the rectal epithelial (REP) cells. b The ratio of NAS-37::GFP expression of L3 and L4 C. elegans was analyzed. $(n>60, * P<0.05)$ a
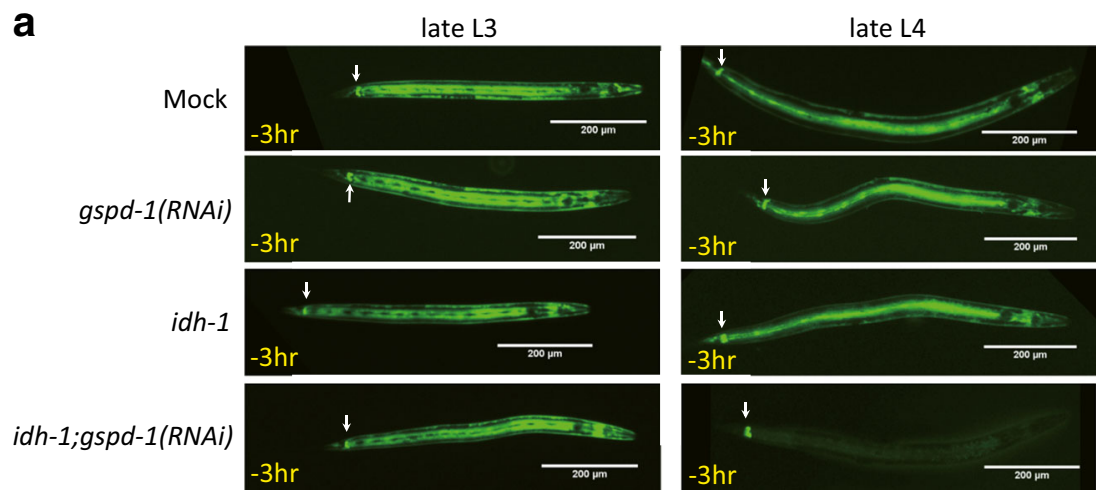

b

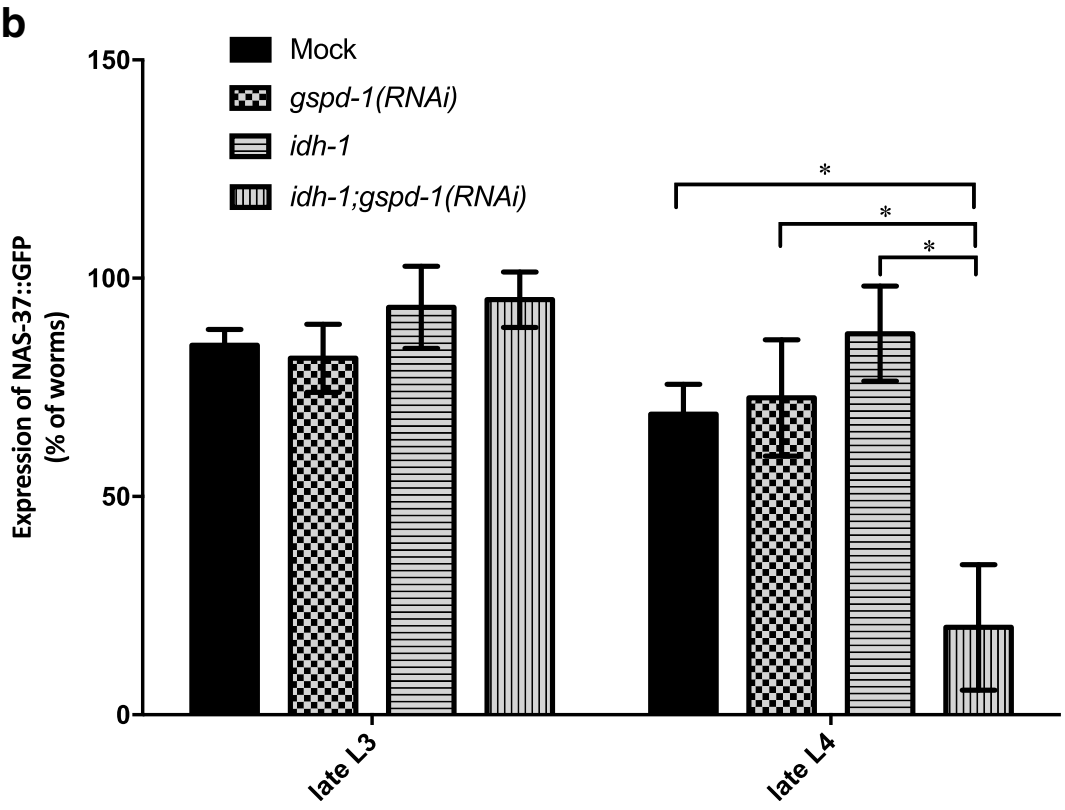

The decrease in amino acids which requires NADPH in their biosynthetic pathways suggests that severe insufficiency of NADPH may affect protein synthesis as illustrated by the impaired molting process in $i d h-1 ; g s p d-1(R N A i)$ doubledeficient $C$. elegans model. C. elegans molting is a tissue remodeling process, which requires the activity of proteases to degrade old cuticle proteins. The status of proteases can lead to molting defects. A decrease in the level of NAS-37 expression in idh-1;gspd-1(RNAi) double-deficient $C$. elegans during the larval stage suggests that the protease is affected by NADPH insufficiency. Nas-37 is expressed in the hypodermis prior to ecdysis at each larval stage in C. elegans [29]. The gene product of nas-37, an Astacin-class metalloprotease, accumulates in the anterior cuticle and is secreted to degrade the old cuticle after ecdysis. A scheme is outlined that describe the effects of $i d h-1 ; g s p d-1(R N A i) 1$ double deficiency in C. elegans (Fig. 6b). NADPH is an important fuel to drive the development machinery. Decreased amino acid synthesis is a main metabolic alteration leading to growth retardation in idh-1;gspd-1(RNAi) double-deficient $C$. elegans. In addition, decreased NAS-37 expression and the molting defect are likely to cause growth retardation through the unshed cuticle restricting the growth of $i d h-1 ; g s p d-1$ (RNAi) double-deficient C. elegans. Solid evidence to prove a causal relationship between NAS-37 expression and impaired amino acid synthesis awaits further investigation.

Currently, there is little information of the relationship between NADPH and NAS-37 or molting in C. elegans. A few studies indicate that a collagen-modifying enzyme, known as NADPH dual oxidase or Bli-3, catalyzes the crosslinking of cuticular collagens $[34,35]$. In the current study, however, the blister phenotype of bli-3 mutant has not been observed in $i d h$ -

Fig. 4 Distinct metabolic alterations of $i d h-1 ; g s p d-1(R N A i)$ doubledeficient C. elegans. a Data were subject to principal component analysis, and the score plots (left panel: ESI positive, right panel: ESI negative; Mock: cyan, gspd-1 deficiency: red, idh-1: blue, idh-1;gspd-1(RNAi) double deficiency: green) were shown. Colored areas represented $95 \%$ confidence regions. b Pathway analysis of datasets indicated potential pathways that were significantly changed in $i d h-1 ; g s p d-1$ (RNA $i$ ) doubledeficient $C$. elegans. The global metabolomic view displays all anabolic and catabolic pathways that are ranked based on scores from pathway topology analysis (X-axis: pathway impact) and from pathway enrichment analysis (Y-axis: $\log (\mathrm{p}))$ 

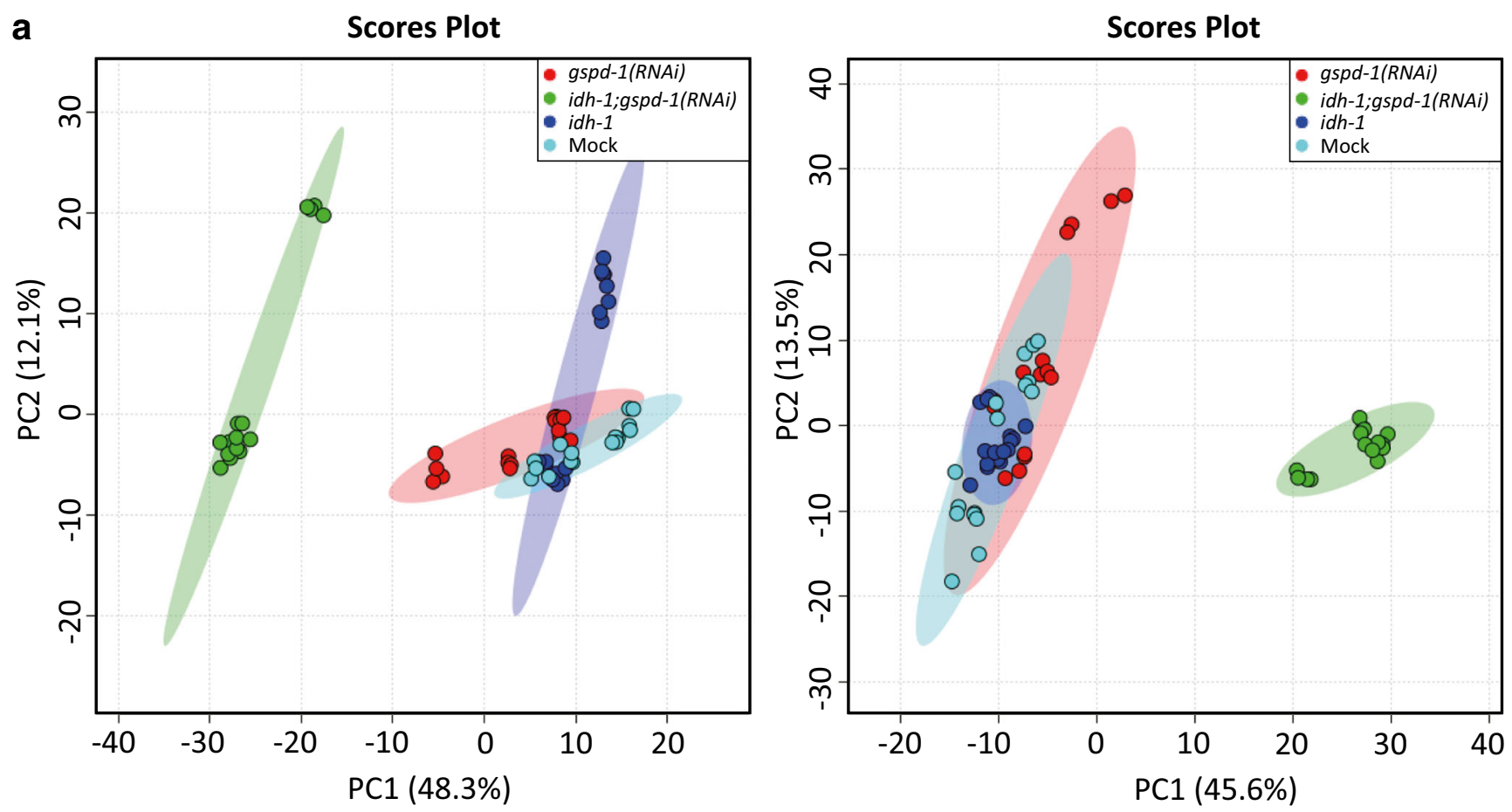

b

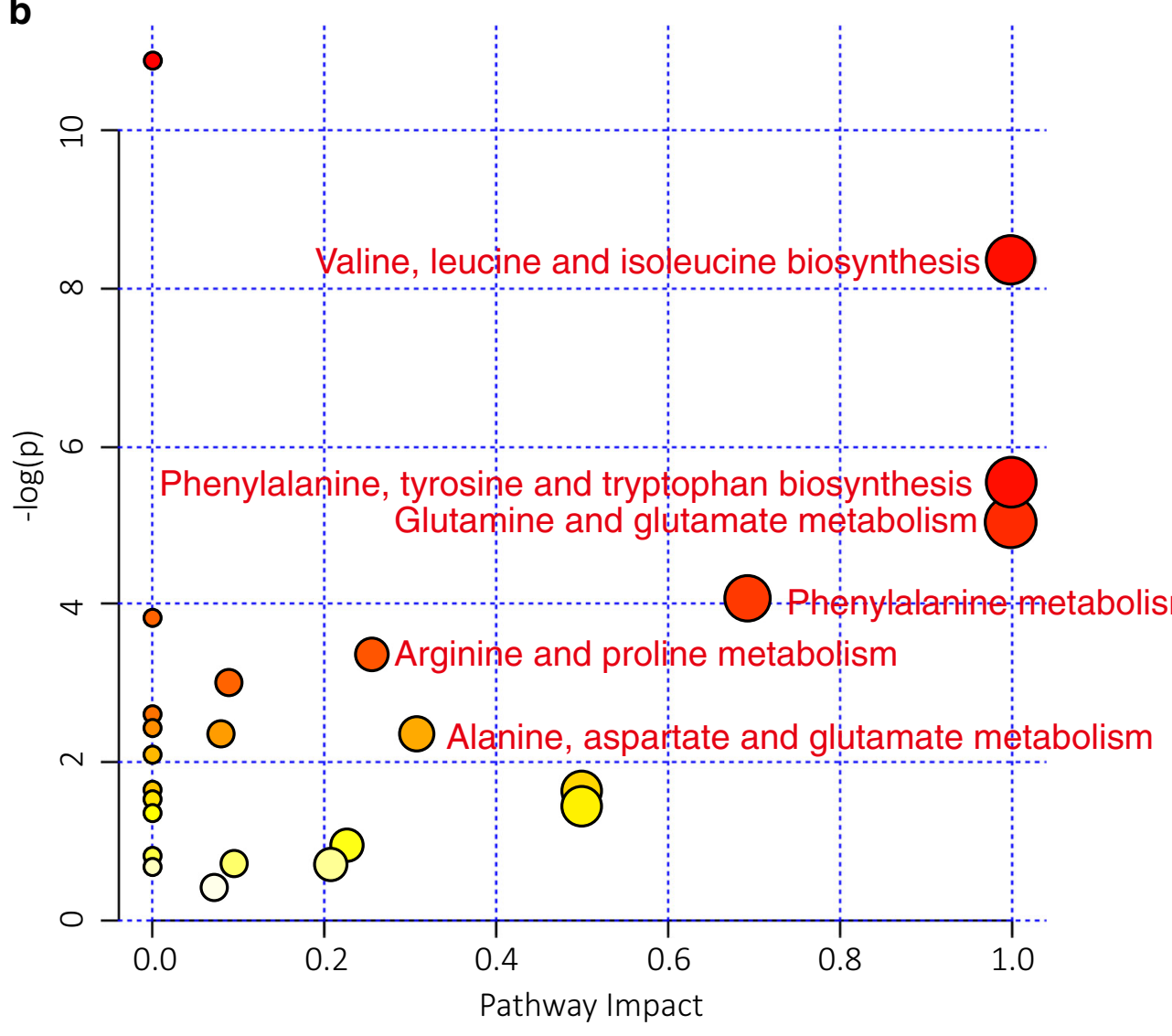


Fig. 5 Altered amino acid metabolism in idh-1;gspdI(RNAi) double-deficient C. elegans. a Glutamine and glutamate metabolism. b Phenylalanine metabolism. c Valine, leucine, and isoleucine biosynthesis. d Arginine and proline metabolism. All data were presented as the mean \pm S.D.. and the statistical difference was analyzed by the two-tailed $t$ test. $(* P<0.05 ; * * P<0.005$; $* * * P<0.001)$ a Glutamine and glutamate metabolism

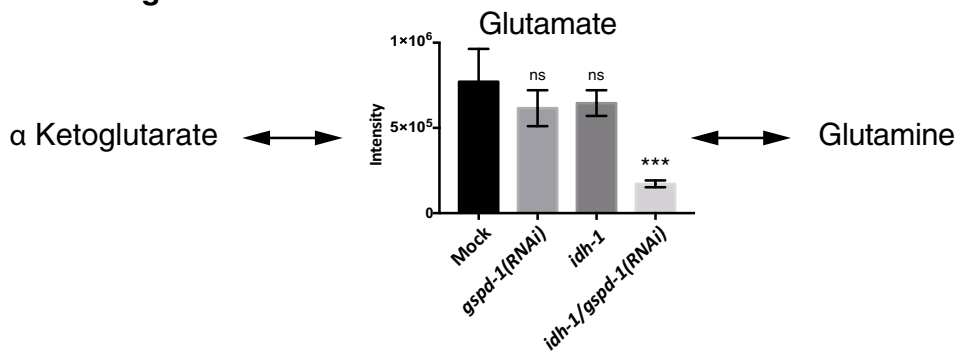

b Phenylalanine, tyrosine and tryptophan biosynthesis

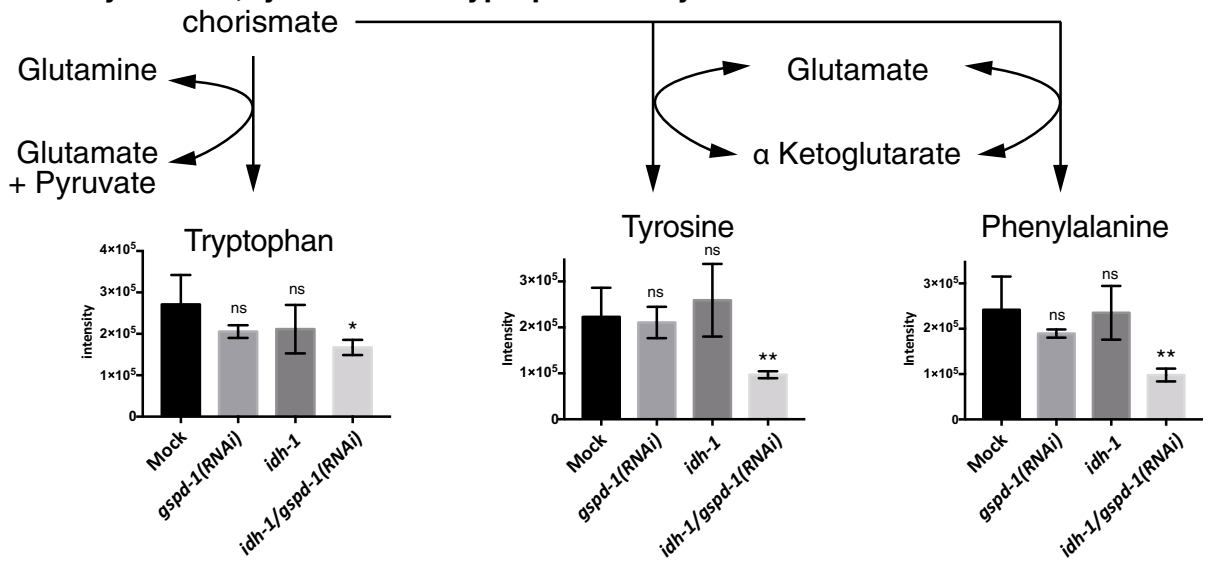

C Valine, leucine and isoleucine biosynthesis

Pyruvate

Oxaloacetate

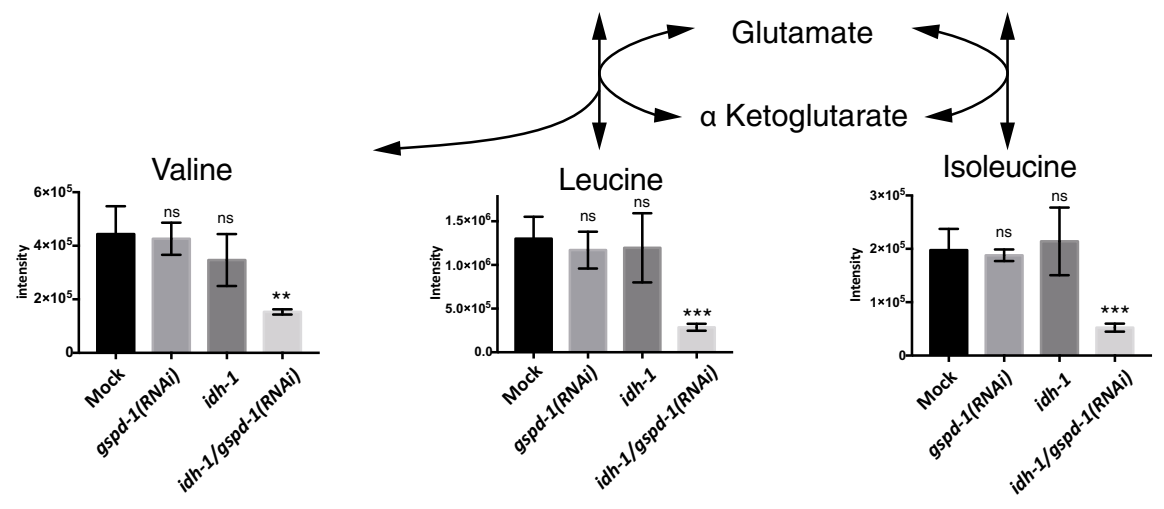

\section{d Arginine and proline metabolism}

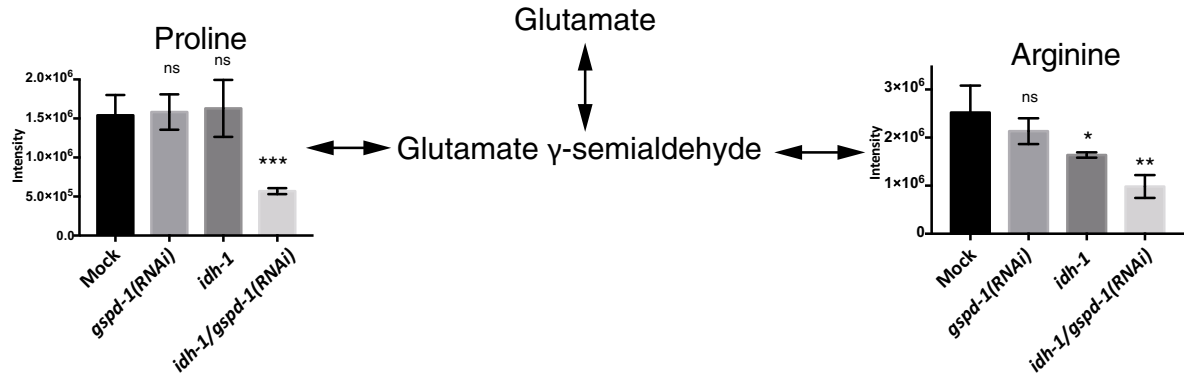

1;gspd-1(RNAi) double-deficient C. elegans. Hence, another yet to be identified target(s) may be modulated by NADPH depletion leading to growth defects in $C$. elegans. Abnormal molting in idh-1;gspd-1(RNAi) double-deficient C. elegans could be, in part, attributed to abnormal fatty acid synthesis because genes of fatty acid synthesis participate in the molting process in C. elegans [36]. Knockdown of fasn-1 and pod-2 by RNAi downregulates the protein expression of NAS-37. 


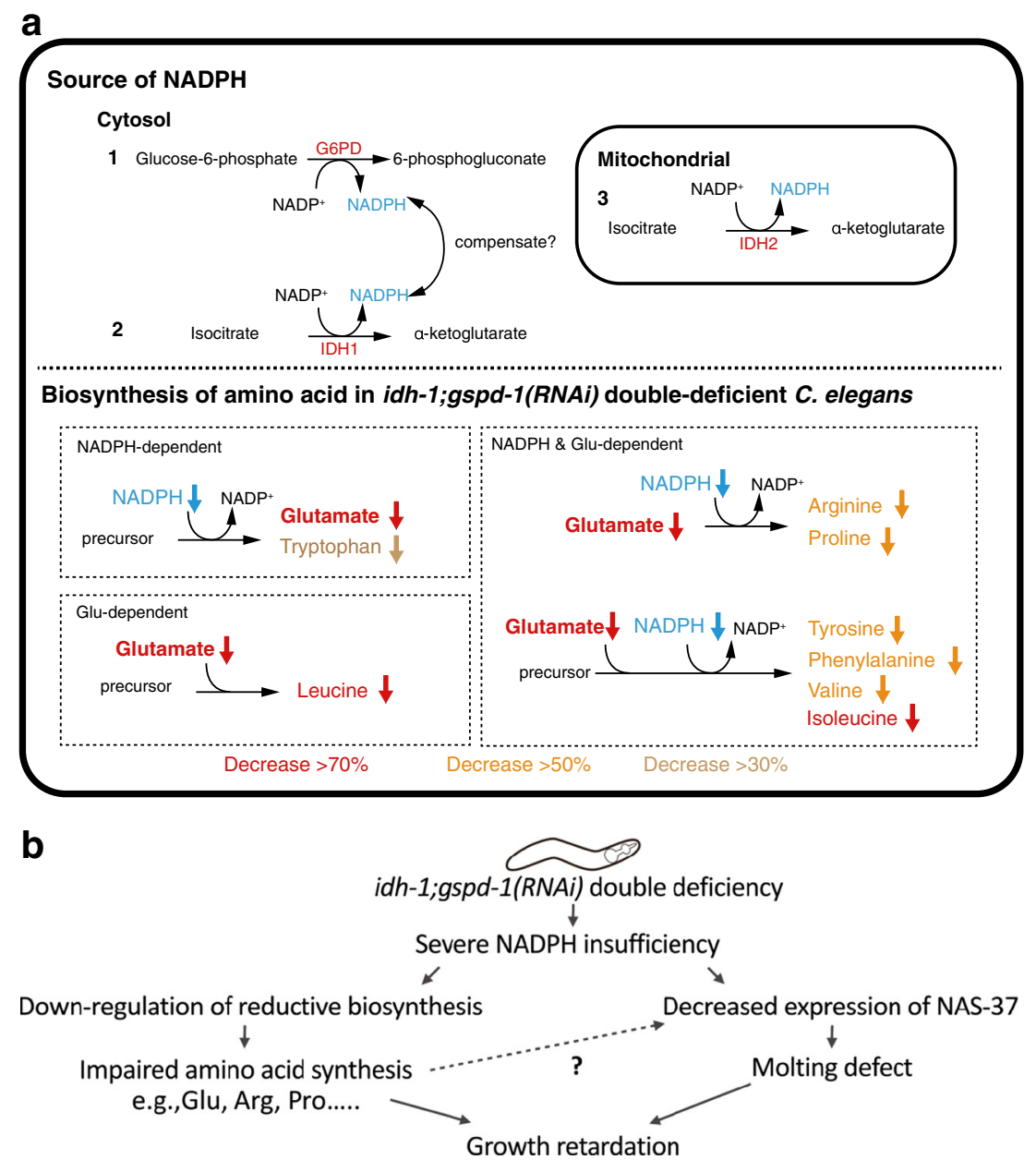

Fig. 6 Proposed schemes for amino acid metabolism as well as for growth and development in $i d h-1 ; g s p d-1(R N A i)$ double-deficient C. elegans. a Upper panel depicts three NADPH producing systems in C. elegans. Both GSPD-1 and IDH-1 are cytosolic enzymes that produce $\mathrm{NADPH}$, whereas IDH-2 is the source of NADPH in mitochondria. Lower panel shows altered amino acid pathways in idh-1;gspd-1(RNAi) double-deficient $C$. elegans. These pathways are classified into three categories based on the requirement of NADPH or glutamate in amino acid synthesis. The decreased levels of amino acids are highlighted in red (>70\%), orange (>50\%), and beige (>30\%). b Hypothetical scheme of the physiological response in idh-1;gspd-1(RNAi) double-deficient C. elegans. The impaired amino acid synthesis may be due to the lack of NADPH for reductive biosynthesis. As a result, defective protein expression fails to support the growth of $C$. elegans. In addition, decreased expression of NAS-37 is linked to the molting defect, ultimately leading to retarded growth
Since NADPH is required for de novo fatty acid synthesis, it is possible that GSPD-1 and IDH-1 double-deficiency-derived NADPH insufficiency disrupts fatty acid biosynthesis leading to NAS-37 inactivation and the molting defect.

G6PD status is associated with many human diseases, including hemolytic disorders, cardiovascular diseases, and diabetes [2]. Although the relationship between G6PD and cancer is unclear, G6PD is involved in transformation and angiogenesis [37]. Overexpression and modification of G6PD promotes tumor growth and leads to a poor clinical outcome [38-43], while suppression of G6PD inhibits cancer development $[42,44-46]$. The notion that G6PD favors rapid cell proliferation is consistent with the finding that inactivation of G6PD causes embryonic lethality $[5,6]$. In short, the current study provides evidence for the involvement of G6PD and IDH1 in cellular as well as organismal growth and development. Similar to G6PD, IDHs play a diverse role in pathophysiology [47-49].

G6PD-derived NADPH is implicated in maintaining redox homeostasis and reductive biosynthesis [3]. Metabolomic studies have demonstrated that G6PD participates in the rapid response of metabolic rerouting to counteract oxidative stress [30, 50]. Lipidomic analysis of embryos derived from GSPD-1deficient $C$. elegans exhibit higher levels of lipid peroxidation [6]. Increased expression of G6PD in the transgenic mice model shows the enhanced NADPH production and reduces oxidative damage [51]. G6PD transgenic mice exhibit improved protection against an age-related functional decline and an extended health span in females. The metabolomic approach employed in the current study provides additional information concerning the complementary role of GSPD-1 and IDH-1 in maintaining cellular redox homeostasis and "redox-regulated biosynthesis." 


\section{Materials and methods}

\section{Nematode culture and RNAi silencing}

N2 (wild type), idh-1(ok2832), and idh-2(ok3184) mutants were acquired from Caenorhabditis Genetics Center (University of Minnesota, Minneapolis, USA). The NAS-37 reporter strain EG3198 was a gift from Prof. Erik Jorgensen (University of Utah, Salt Lake City, USA). The strains were maintained at $20^{\circ} \mathrm{C}$ on Nematode Growth Medium (NGM) agar plate seeded with live E. coli OP50 bacterial lawn based on standard protocols [52]. The gspd-1-RNAi silencing experiment was performed as described previously $[5,6]$. The $i d h-1$ and $g s p d-1$ double-deficient strain was created by using $g s p d-$ 1 RNAi in idh-1(ok2832) background. The idh-2 and gspd-1 double-deficient strain was created by using $g s p d-1$ RNAi in idh-2(ok3184) background. The RNAi strains, using live E. coli $H T 115(D E 3)$ as a food source, were maintained at $20{ }^{\circ} \mathrm{C}$ on NGM agar plate supplemented with $1 \mathrm{mM}$ IPTG.

\section{Phenotype assays}

For the molting assay, synchronized $\mathrm{L} 1$ grown at $20^{\circ} \mathrm{C}$ for $48 \mathrm{~h}$ was picked and mounted on a $2 \%$ agarose pad on a glass slide followed by anesthetizing with $0.2 \%$ levamisole, and a cover slide was placed on the agarose pad. DIC and fluorescent images were taken by using an epifluorescence microscope (Leica, Wetzlar, Germany). For the body size and locomotion assays, synchronized L1 was cultivated on NGM plates at $20^{\circ} \mathrm{C}$ for $72 \mathrm{~h}$. Images and video clips were taken by using a dissecting microscope (Nikon, Japan) with a MoticCam X CMOS camera (Motic, Xiamen, China) followed by image analysis (Metamorph 6.1r0; Molecular Device, CA, USA).

\section{Metabolomic analysis}

The synchronized L1 worms were cultured at $20{ }^{\circ} \mathrm{C}$ until adulthood. Four biological replicates of adult worms were washed off NGM plates by ultra-water. The samples were washed twice by M9 buffer and were centrifuged at $2500 \mathrm{rpm}$ for $1 \mathrm{~min}$. The samples were suspended with $80 \%$ methanol and transferred to a homogenization tube pre-filled with 1.0-mm diameter Zirconia beads (Biospec, Bartlesville, OK, USA) and were homogenized in a Precellys 24 homogenizer coupled with a Cryolys Cooling System (Bertin Instrument, Rockville, MD, USA). The homogenization was set at two cycles of $6500 \mathrm{rpm}$ for $30 \mathrm{~s}$ with an interval of $5 \mathrm{~s}$. Subsequently, the samples were centrifuged at $12000 \mathrm{rpm}$ for 15 min at $4{ }^{\circ} \mathrm{C}$. The supernatant of each sample was transferred to a separate glass tube. The homogenization tube was refilled with $1 \mathrm{ml}$ of $80 \%$ methanol followed by additional homogenization and centrifugation to recover residual samples. The supernatant was transferred to the previous glass tube and air dried under nitrogen flow in a nitrogen evaporator (Taitec, Koshigaya-shi, Saitama-ken, Japan) and stored at $80{ }^{\circ} \mathrm{C}$. The pellet was dissolved in $1 \mathrm{ml}$ of $0.1 \mathrm{~N} \mathrm{NaOH}$ at $65{ }^{\circ} \mathrm{C}$ for $30 \mathrm{~min}$ to determine the protein concentration (Bradford assay).

Prior to metabolomic analysis, the sample was dissolved in $300 \mu \mathrm{l}$ LC-MS Chromasolv water (Fluka) containing $0.1 \%$ formic acid. The tube was vortexed for $30 \mathrm{~s}$ and repeated four times. The mixture was transferred to an eppendorf tube and centrifuged at $12000 \mathrm{rpm}$ for $30 \mathrm{~min}$ at $4{ }^{\circ} \mathrm{C}$. The supernatant was then transferred to an HPLC vial for liquid chromatography and mass spectrometry analysis. For LC-MS analysis, mass spectrometry analysis was carried out using an Agilent 1200 rapid resolution liquid chromatography system coupled with an Agilent 6510 Q-TOF MS system (Agilent Technologies, CA, USA), which is equipped with an electrospray ionization source. Chromatographic separation was performed on an Acquity UPLC HSST3 reversed phase C18 column (particle size of $1.8 \mu \mathrm{m}, 2.1 \mathrm{~mm} \times 150 \mathrm{~mm}$ ) (Waters, Milford, USA). Column temperature was maintained at $40{ }^{\circ} \mathrm{C}$ and the flow rate was $0.25 \mathrm{ml} / \mathrm{min}$. For metabolite profiling, the mobile phase consisted of $0.1 \%$ formic acid (solvent $\mathrm{A}$ ) and $0.1 \%$ formic acid/acetonitrile (solvent $\mathrm{B}$ ). The mobile phase condition was listed below: solvent $\mathrm{A}$, 2 min; gradient from 0 to $40 \%$ solvent B, 4 min; $40 \%$ solvent B, 2 min; gradient from 40 to $98 \%$ solvent B, 2 min; $98 \%$ solvent B, 6 min; gradient from 98 to $0 \%$ solvent B, 2 min.

The samples were subjected to RRLC-ESI-TOF-MS. Mass spectrometric analysis was performed in the ESI positive and ESI negative modes. The pressure of the nitrogen nebulizer was set at $30 \mathrm{psi}$ and the nitrogen drying gas was set at $350^{\circ} \mathrm{C}$ with a flow rate of $10 \mathrm{l} / \mathrm{min}$. The skimmer and capillary voltages were set at $65 \mathrm{~V}$ and $4000 \mathrm{~V}$, respectively. Data were obtained over the range from $\mathrm{m} / \mathrm{z} 50$ to $\mathrm{m} / \mathrm{z} 1000$ at a rate of 1 scan per second. Data were collected in the profile mode using Agilent MassHunter Workstation Data acquisition software. For processing data, individual components, or called molecular features, in the sample were identified using the Molecular Feature Extraction (MFE) algorithm of MassHunter software. Upon processing, the raw data generated time-aligned ion features (isotopes, adducts, and dimers), the monoisotopic neutral mass, retention time, and ion abundance for each molecular feature. An Agilent GeneSpring-MS (Agilent Technologies, CA, USA) was used to visualize datasets in numerical data matrices (metabolite concentrations). MetaboAnalyst 3.0 was used for analysis and visualization of MS data sets in data matrices and principal components analysis (PCA) diagrams as well as multivariate data analysis and data representation, such as a volcano plot. PCA was employed for clustering and correlation analyses. Relative concentrations of metabolites were compared by ANOVA with a Tukey HSD correction. Accurate masses of 
features showing significant differences between control and test groups were searched against our in-house and public metabolite databases, including HMDB (http://www.hmdb. ca), METLIN (http://metlin.scripps.edu/index.php), and KEGG (http://www.genome.jp/kegg/).

\section{Statistical analysis}

Where applicable, all data were shown as the mean \pm S.D. The statistical difference was analyzed by the two-tailed $t$ test. All statistical tests were conducted using the GraphPad Prism 6.0 (San Diego, CA, USA). Values of $P<0.05$ were considered statistically significant.

Acknowledgements The reporter strain EG3198 was kindly provided by Prof. Erik Jorgensen (University of Utah, Salt Lake City, USA).

Funding information This article is made possible by grants from the Ministry of Science and Technology of Taiwan (MOST105-2320-B-182031-MY2, MOST107-2320-B-182-013 to DTYC, and MOST107-2320B-264-001-MY2 to HCY) and from Chang Gung Memorial Hospital (BMRP098, CMRPD1F0462, CMRPD1F0622 to DTYC)

Open Access This article is distributed under the terms of the Creative Commons Attribution 4.0 International License (http:// creativecommons.org/licenses/by/4.0/), which permits unrestricted use, distribution, and reproduction in any medium, provided you give appropriate credit to the original author(s) and the source, provide a link to the Creative Commons license, and indicate if changes were made.

Publisher's Note Springer Nature remains neutral with regard to jurisdictional claims in published maps and institutional affiliations.

\section{References}

1. Beutler E (1994) G6PD deficiency. Blood 84:3613-3636

2. Ho HY, Cheng ML, Chiu DT (2014) Glucose-6-phosphate dehydrogenase-beyond the realm of red cell biology. Free Radic Res 48: $1028-1048$

3. Yang HC, Wu YH, Liu HY, Stern A, Chiu DT (2016) What has passed is prolog: new cellular and physiological roles of G6PD. Free Radic Res 50:1047-1064

4. Longo L, Vanegas OC, Patel M, Rosti V, Li H, Waka J, Merghoub T, Pandolfi PP, Notaro R, Manova K, Luzzatto L (2002) Maternally transmitted severe glucose 6-phosphate dehydrogenase deficiency is an embryonic lethal. EMBO J 21:4229-4239

5. Yang HC, Chen TL, Wu YH, Cheng KP, Lin YH, Cheng ML, Ho HY, Lo SJ, Chiu DT (2013) Glucose 6-phosphate dehydrogenase deficiency enhances germ cell apoptosis and causes defective embryogenesis in Caenorhabditis elegans. Cell Death Dis 4:e616

6. Chen TL, Yang HC, Hung CY, Ou MH, Pan YY, Cheng ML, Stern A, Lo SJ, Chiu DT (2017) Impaired embryonic development in glucose-6-phosphate dehydrogenase-deficient Caenorhabditis elegans due to abnormal redox homeostasis induced activation of calcium-independent phospholipase and alteration of glycerophospholipid metabolism. Cell Death Dis 8:e2545
7. Patrinostro X, Carter ML, Kramer AC, Lund TC (2013) A model of glucose-6-phosphate dehydrogenase deficiency in the zebrafish. Exp Hematol 41:697-710.e692

8. Lewis CA, Parker SJ, Fiske BP, McCloskey D, Gui DY, Green CR, Vokes NI, Feist AM, Vander Heiden MG, Metallo CM (2014) Tracing compartmentalized NADPH metabolism in the cytosol and mitochondria of mammalian cells. Mol Cell 55:253-263

9. Fan J, Ye J, Kamphorst JJ, Shlomi T, Thompson CB, Rabinowitz JD (2014) Quantitative flux analysis reveals folate-dependent NADPH production. Nature 510:298-302

10. Reitman ZJ, Yan H (2010) Isocitrate dehydrogenase 1 and 2 mutations in cancer: alterations at a crossroads of cellular metabolism. J Natl Cancer Inst 102:932-941

11. Ronnebaum SM, Ilkayeva O, Burgess SC, Joseph JW, Lu D, Stevens RD, Becker TC, Sherry AD, Newgard CB, Jensen MV (2006) A pyruvate cycling pathway involving cytosolic NADPdependent isocitrate dehydrogenase regulates glucose-stimulated insulin secretion. J Biol Chem 281:30593-30602

12. Koh HJ, Lee SM, Son BG, Lee SH, Ryoo ZY, Chang KT, Park JW, Park DC, Song BJ, Veech RL, Song H, Huh TL (2004) Cytosolic NADP+-dependent isocitrate dehydrogenase plays a key role in lipid metabolism. J Biol Chem 279:39968-39974

13. Comte B, Vincent G, Bouchard B, Benderdour M, Des Rosiers C (2002) Reverse flux through cardiac NADP(+)-isocitrate dehydrogenase under normoxia and ischemia. Am J Physiol Heart Circ Physiol 283:H1505-H1514

14. Nekrutenko A, Hillis DM, Patton JC, Bradley RD, Baker RJ (1998) Cytosolic isocitrate dehydrogenase in humans, mice, and voles and phylogenetic analysis of the enzyme family. Mol Biol Evol 15: 1674-1684

15. Cairns RA, Mak TW (2013) Oncogenic isocitrate dehydrogenase mutations: mechanisms, models, and clinical opportunities. Cell Biosci 3:730-741

16. Ward PS, Patel J, Wise DR, Abdel-Wahab O, Bennett BD, Coller HA, Cross JR, Fantin VR, Hedvat CV, Perl AE, Rabinowitz JD, Carroll M, Su SM, Sharp KA, Levine RL, Thompson CB (2010) The common feature of leukemia-associated IDH1 and IDH2 mutations is a neomorphic enzyme activity converting alphaketoglutarate to 2-hydroxyglutarate. Cancer Cell 17:225-234

17. Itsumi M, Inoue S, Elia AJ, Murakami K, Sasaki M, Lind EF, Brenner D, Harris IS, Chio II, Afzal S et al (2015) Idh1 protects murine hepatocytes from endotoxin-induced oxidative stress by regulating the intracellular NADP(+)/NADPH ratio. Cell Death Differ 22:1837-1845

18. Ye J, Gu Y, Zhang F, Zhao Y, Yuan Y, Hao Z, Sheng Y, Li WY, Wakeham A, Cairns RA, Mak TW (2017) IDH1 deficiency attenuates gluconeogenesis in mouse liver by impairing amino acid utilization. Proc Natl Acad Sci U S A 114:292-297

19. Ho HY, Cheng ML, Lu FJ, Chou YH, Stern A, Liang CM, Chiu DT (2000) Enhanced oxidative stress and accelerated cellular senescence in glucose-6-phosphate dehydrogenase (G6PD)-deficient human fibroblasts. Free Radic Biol Med 29:156-169

20. Minard KI, Carroll CA, Weintraub ST, Mc-Alister-Henn L (2007) Changes in disulfide bond content of proteins in a yeast strain lacking major sources of NADPH. Free Radic Biol Med 42:106-117

21. Braeckman BP, Houthoofd K, Vanfleteren JR (2009) Intermediary metabolism. WormBook: 1-24. DOI https://doi.org/10.1895/ wormbook.1.146.1

22. Atherton HJ, Jones OA, Malik S, Miska EA, Griffin JL (2008) A comparative metabolomic study of NHR-49 in Caenorhabditis elegans and PPAR-alpha in the mouse. FEBS Lett 582:1661-1666

23. Hughes SL, Bundy JG, Want EJ, Kille P, Sturzenbaum SR (2009) The metabolomic responses of Caenorhabditis elegans to cadmium are largely independent of metallothionein status, but dominated by changes in cystathionine and phytochelatins. J Proteome Res 8: 3512-3519 
24. Castro C, Sar F, Shaw WR, Mishima M, Miska EA, Griffin JL (2012) A metabolomic strategy defines the regulation of lipid content and global metabolism by Delta9 desaturases in Caenorhabditis elegans. BMC Genomics 13:36

25. Patti GJ, Tautenhahn R, Johannsen D, Kalisiak E, Ravussin E, Bruning JC, Dillin A, Siuzdak G (2014) Meta-analysis of global metabolomic data identifies metabolites associated with life-span extension. Metabolomics 10:737-743

26. Morgan PG, Higdon R, Kolker N, Bauman AT, Ilkayeva O, Newgard CB, Kolker E, Steele LM, Sedensky MM (2015) Comparison of proteomic and metabolomic profiles of mutants of the mitochondrial respiratory chain in Caenorhabditis elegans. Mitochondrion 20:95-102

27. Wang W, McReynolds MR, Goncalves JF, Shu M, Dhondt I, Braeckman BP, Lange SE, Kho K, Detwiler AC, Pacella MJ et al (2015) Comparative metabolomic profiling reveals that dysregulated glycolysis stemming from lack of salvage NAD+ biosynthesis impairs reproductive development in Caenorhabditis elegans. J Biol Chem 290:26163-26179

28. Wan QL, Shi X, Liu J, Ding AJ, Pu YZ, Li Z, Wu GS, Luo HR (2017) Metabolomic signature associated with reproductionregulated aging in Caenorhabditis elegans. Aging (Albany NY) 9:447-474

29. Davis MW, Birnie AJ, Chan AC, Page AP, Jorgensen EM (2004) A conserved metalloprotease mediates ecdysis in Caenorhabditis elegans. Development 131:6001-6008

30. Tang HY, Ho HY, Wu PR, Chen SH, Kuypers FA, Cheng ML, Chiu DT (2015) Inability to maintain GSH pool in G6PD-deficient red cells causes futile AMPK activation and irreversible metabolic disturbance. Antioxid Redox Signal 22:744-759

31. Cheng ML, Shiao MS, Chiu DT, Weng SF, Tang HY, Ho HY (2011) Biochemical disorders associated with antiproliferative effect of dehydroepiandrosterone in hepatoma cells as revealed by LC-based metabolomics. Biochem Pharmacol 82:1549-1561

32. Penkov S, Kaptan D, Erkut C, Sarov M, Mende F, Kurzchalia TV (2015) Integration of carbohydrate metabolism and redox state controls dauer larva formation in Caenorhabditis elegans. Nat Commun 6:8060

33. Szewczyk NJ, Kozak E, Conley CA (2003) Chemically defined medium and Caenorhabditis elegans. Bmc Biotechnol 3. DOI Artn 19. Doi https://doi.org/10.1186/1472-6750-3-19

34. Thein MC, Winter AD, Stepek G, McCormack G, Stapleton G, Johnstone IL, Page AP (2009) Combined extracellular matrix cross-linking activity of the peroxidase MLT-7 and the dual oxidase BLI-3 is critical for post-embryonic viability in Caenorhabditis elegans. J Biol Chem 284:17549-17563

35. Moribe H, Konakawa R, Koga D, Ushiki T, Nakamura K, Mekada E (2012) Tetraspanin is required for generation of reactive oxygen species by the dual oxidase system in Caenorhabditis elegans. PLoS Genet 8:e1002957

36. Li Y, Paik YK (2011) A potential role for fatty acid biosynthesis genes during molting and cuticle formation in Caenorhabditis elegans. BMB Rep 44:285-290

37. Leopold JA, Walker J, Scribner AW, Voetsch B, Zhang YY, Loscalzo AJ, Stanton RC, Loscalzo J (2003) Glucose-6phosphate dehydrogenase modulates vascular endothelial growth factor-mediated angiogenesis. J Biol Chem 278:32100-32106
38. Pu H, Zhang Q, Zhao C, Shi L, Wang Y, Wang J, Zhang M (2015) Overexpression of G6PD is associated with high risks of recurrent metastasis and poor progression-free survival in primary breast carcinoma. World J Surg Oncol 13:323

39. Wang J, Yuan W, Chen Z, Wu S, Chen J, Ge J, Hou F, Chen Z (2012) Overexpression of G6PD is associated with poor clinical outcome in gastric cancer. Tumour Biol 33:95-101

40. Rao X, Duan X, Mao W, Li X, Li Z, Li Q, Zheng Z, Xu H, Chen M, Wang PG, Wang Y, Shen B, Yi W (2015) O-GlcNAcylation of G6PD promotes the pentose phosphate pathway and tumor growth. Nat Commun 6:8468

41. Kowalik MA, Guzzo G, Morandi A, Perra A, Menegon S, Masgras I, Trevisan E, Angioni MM, Fornari F, Quagliata L, LeddaColumbano GM, Gramantieri L, Terracciano L, Giordano S, Chiarugi P, Rasola A, Columbano A (2016) Metabolic reprogramming identifies the most aggressive lesions at early phases of hepatic carcinogenesis. Oncotarget 7:32375-32393

42. Xu SN, Wang TS, Li X, Wang YP (2016) SIRT2 activates G6PD to enhance NADPH production and promote leukaemia cell proliferation. Sci Rep 6:32734

43. Dore MP, Davoli A, Longo N, Marras G, Pes GM (2016) Glucose6-phosphate dehydrogenase deficiency and risk of colorectal cancer in Northern Sardinia: a retrospective observational study. Medicine (Baltimore) 95:e5254

44. Chen Y, Xu Q, Ji D, Wei Y, Chen H, Li T, Wan B, Yuan L, Huang R, Chen $G$ (2016) Inhibition of pentose phosphate pathway suppresses acute myelogenous leukemia. Tumour Biol 37:6027-6034

45. Gregory MA, D'Alessandro A, Alvarez-Calderon F, Kim J, Nemkov T, Adane B, Rozhok AI, Kumar A, Kumar V, Pollyea DA et al (2016) ATM/G6PD-driven redox metabolism promotes FLT3 inhibitor resistance in acute myeloid leukemia. Proc Natl Acad Sci U S A 113:E6669-E6678

46. Ju HQ, Lu YX, Wu QN, Liu J, Zeng ZL, Mo HY, Chen Y, Tian T, Wang Y, Kang TB, Xie D, Zeng MS, Huang P, Xu RH (2017) Disrupting G6PD-mediated redox homeostasis enhances chemosensitivity in colorectal cancer. Oncogene 36:6282-6292

47. Waitkus MS, Diplas BH, Yan H (2016) Isocitrate dehydrogenase mutations in gliomas. Neuro-Oncology 18:16-26

48. Medeiros BC, Fathi AT, DiNardo CD, Pollyea DA, Chan SM, Swords R (2017) Isocitrate dehydrogenase mutations in myeloid malignancies. Leukemia 31:272-281

49. Al-Khallaf H (2017) Isocitrate dehydrogenases in physiology and cancer: biochemical and molecular insight. Cancer Discov 7:37

50. Kuehne A, Emmert H, Soehle J, Winnefeld M, Fischer F, Wenck H, Gallinat S, Terstegen L, Lucius R, Hildebrand J, Zamboni N (2015) Acute activation of oxidative pentose phosphate pathway as firstline response to oxidative stress in human skin cells. Mol Cell 59: 359-371

51. Nobrega-Pereira S, Fernandez-Marcos PJ, Brioche T, GomezCabrera MC, Salvador-Pascual A, Flores JM, Vina J, Serrano M (2016) G6PD protects from oxidative damage and improves healthspan in mice. Nat Commun 7:10894

52. Girard LR, Fiedler TJ, Harris TW, Carvalho F, Antoshechkin I, Han M, Sternberg PW, Stein LD, Chalfie M (2007) WormBook: the online review of Caenorhabditis elegans biology. Nucleic Acids Res 35:D472-D475 